AperTO - Archivio Istituzionale Open Access dell'Università di Torino

Graphene oxide-mediated electrochemistry of glucose oxidase on glassy carbon electrodes

This is a pre print version of the following article:

Original Citation:

Availability:

This version is available http://hdl.handle.net/2318/1528397

since 2019-03-12T14:03:35Z

Published version:

DOI:10.1002/bab.1392

Terms of use:

Open Access

Anyone can freely access the full text of works made available as "Open Access". Works made available under a Creative Commons license can be used according to the terms and conditions of said license. Use of all other works requires consent of the right holder (author or publisher) if not exempted from copyright protection by the applicable law. 


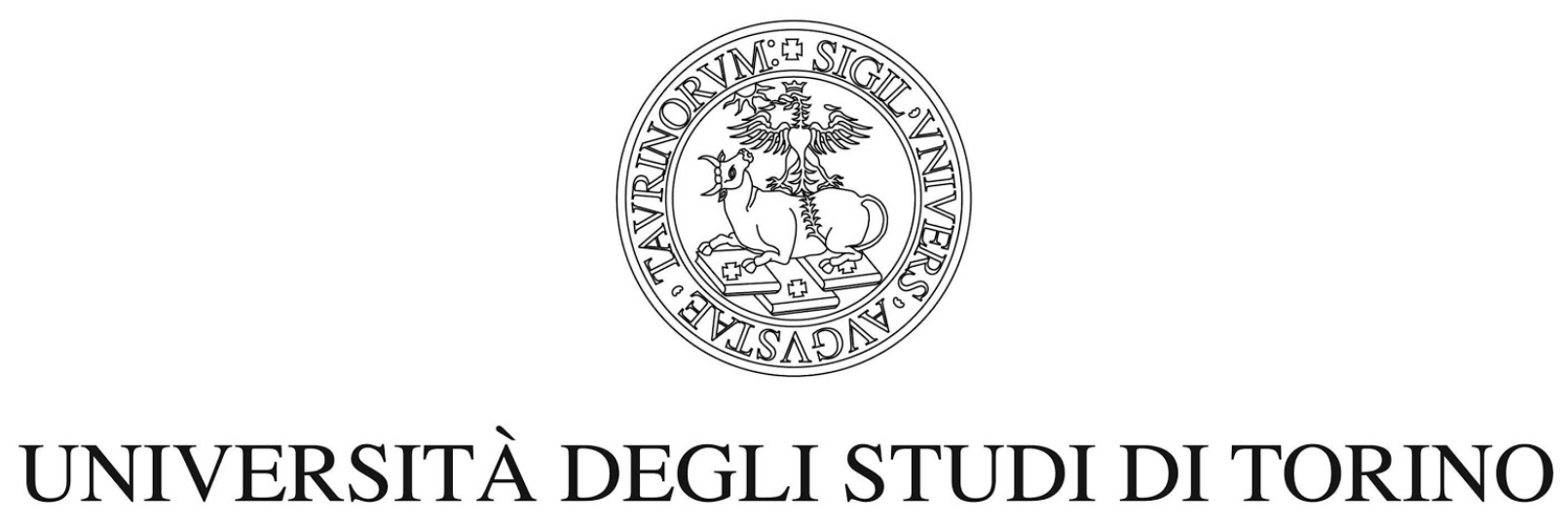

This is the accepted version of the following article:

[Castrignano' S, Valetti F, Gilardi G, Sadeghi SJ. Graphene oxide-mediated electrochemistry of glucose oxidase on glassy carbon electrodes. BAB (2016) 63: 157-162.],

which has been published in final form at [http://onlinelibrary.wiley.com/doi/10.1002/bab.1392/full] 


\section{Graphene oxide mediated electrochemistry of glucose oxidase on glassy carbon electrodes}

Silvia Castrignanò ${ }^{1}$, Francesca Valetti ${ }^{1}$, Gianfranco Gilardi ${ }^{1,2}$ and Sheila J. Sadeghi ${ }^{1,2}{ }^{*}$

${ }^{1}$ Department of Life Sciences and Systems Biology, University of Torino, via Accademia Albertina 13, 10123 Torino, Italy.

${ }^{2}$ Centre for Nanostructured Interfaces and Surfaces, University of Torino, via Pietro Giuria 7, 10125 Torino, Italy.

*Corresponding author: S.J. Sadeghi, Department of Life Sciences and Systems Biology, Via Accademia Albertina 13, 10123 Torino, Italy.

Phone: +39-011-6704528

Fax: +39-011-6704643

e-mail: sheila.sadeghi@unito.it

Running title: Glucose oxidase electrochemistry with graphene oxide 


\begin{abstract}
Glucose oxidase (GOD) was immobilized on glassy carbon electrodes in the presence of graphene oxide (GO) as a model system for the interaction between GO and biological molecules. Lyotropic properties of didodecyldimethylammonium bromide (DDAB) were used to stabilize the enzymatic layer on the electrode surface resulting in a markedly improved electrochemical response of the immobilized GOD. TEM images of the GO with DDAB confirmed the distribution of the GO in a two-dimensional as a foil-like material. Although it is known that glassy carbon surfaces are not ideal for hydrogen peroxide detection, successful chronoamperometric titrations of the GOD in the presence of GO with $\beta$-D-glucose were performed on glassy carbon electrodes whereas no current response was detected upon $\beta$-D-glucose addition in the absence of GO. The GOD-DDAB-GO system displayed a high turnover efficiency and substrate affinity as a glucose biosensor. The simplicity and ease of the electrode preparation procedure of this GO/DDAB system makes it a good candidate for immobilizing other biomolecules for fabrication of amperometric biosensors.
\end{abstract}

\title{
Keywords
}

Graphene oxide, glucose oxidase, glassy carbon, glucose biosensor

\begin{abstract}
Abbreviations
CAT: catalase; DDAB: didodecyldimethylammonium bromide; FAD: flavin adenine dinucleotide; GO: graphene oxide; GOD: glucose oxidase; HRP: horseradish peroxidase; TEM: transmission electron microscopy.
\end{abstract}




\section{Introduction}

Owing to its unique electrical, mechanical and thermal properties, the importance of graphene has been rapidly growing in the recent years, both from a scientific and a commercial point of view. Among graphene derivatives, graphene oxide (GO) plays a major role in the development of carbon nanomaterials. GO synthesis can be achieved by complete exfoliation of graphite oxide, leading to a single graphene sheet extensively functionalized [1]. Unlike graphene structure that consists only of $\mathrm{sp}^{2}$ hybridized carbon atoms, GO carbon structure is interspersed by a variety of oxygen-containing functional groups resulting in a certain degree of $\mathrm{sp}^{3}$ hybridization. The most supported model describing the type and distribution of oxygen functionalities is the one proposed by Lerf and colleagues $[2,3]$ in which the basal plane of the carbon sheet binds hydroxy- and epoxy- functional groups. Carbonyl groups are mostly present as carboxylic groups at the boundary of the sheet [4]. The presence of large amounts of oxygen functional groups is favorable for GO electrochemical applications $[5,6]$ and is thought to support the electron-transfer between electrode substrates and the bio-molecules for electrochemical studies [7].

GO also has functional properties useful for a number of novel electronic, sensing- and energyrelated applications. While GO conductivity is impaired by the oxygen-containing groups, these same groups allow GO to be suspended in water and therefore make it available for a variety of chemical reactions. Most importantly, solubility in aqueous solutions also makes GO compatible with biological applications not available to graphene since it is insoluble in water and requires the support of a substrate $[8,9]$.

Due to its attractive qualities, graphene has also been used as a transducer to develop a number of biosensing schemes: bio-field-effect transistors, electrochemical biosensors, impedance biosensors, electro chemiluminescence, and fluorescence biosensors, as well as biomolecular labels [10-12]. Amongst a variety of biological applications, such as in vivo and in vitro imaging and drug delivery, GO-based biosensors have also been developed and described [13-15]. The metal-free, large surface 
area of GO is highly sensitive to electrochemical changes in the immediate surroundings. The chemical response seems to be a result of the charge transfer between GO and the adsorbed biomolecules. When molecules adsorb on GO, a charge transfer occurs on the site of adsorption involving $\mathrm{GO}$ as a donor or acceptor, changing the carrier density and electrical resistance of GO [16]. This enables its use in various chemical sensors.

GO has also proven to possess a great electrocatalytic activity toward hydrogen peroxide [17] and this capability has been attributable to high density, edge-plane-like defective sites on GO which provide several active sites for electron transfer to biological species [18].

When developing GO based bio-analytical platforms, a key role is played by the stabilization of the bio-molecule as well as the preservation of its native structure and activity. Particularly, this is essential in the case of enzyme based electrochemical systems in which the catalytic activity is strongly dependent on the correct fold of the protein molecule. In this regard, the use of a stabilizing component that could support enzyme structure and activity together with the electrochemical interaction between the bio-component and the GO based transducer can be greatly advantageous. To this end, surfactant films of synthetic lipids such as didodecyldimethylammonium bromide (DDAB) can provide a biomembrane-like microenvironment containing enough water for supporting the structure and activity of proteins on electrode surfaces by acting as stable lyotropic liquid crystal coats [19]. This surfactant has been successfully employed for electrochemical studies of human monooxygenases by our group [20,21].

Herein, the capability of GO to act as an electron relay between the electrode surface and the DDAB immobilized redox enzymes, glucose oxidase (GOD) was chosen as a model enzyme and the direct electron transfer of the immobilized GOD was investigated. Moreover, the electrocatalytic activity of the GOD electrode system was investigated by detection of $\beta$-D-glucose using chronoamperometry. GO has already shown to improve both direct electrochemistry and electrocatalytic activity of redox proteins [22,23] and has been defined as an excellent electrode 
material for GOD based biosensors [17,24,25]. Glucose sensors impact in the routine clinical screening tests is nowadays very high. The number of glucose assays used for diabetic tests exceeds ten million, and glucose biosensors represent about $85 \%$ of the entire sensing market [24].

Direct electrochemistry of GOD in the presence of DDAB-GO was studied by cyclic voltammetry and the detectability of $\beta$-D-glucose on GOD-DDAB-GO electrodes was carried out using chronoamperometric titration. The obtained GO based GOD sensor was also characterized by $\beta$-Dglucose detection limits and reproducibility.

\section{Experimental}

\subsection{Chemicals}

GO (4 mg/mL, water dispersion) was purchased from Graphenea (Spain). Analytical grade chemicals were used with no further purification. All solutions were prepared with ultra pure deionized water. Glucose oxidase (GOD type VII from Aspergillus niger, EC 1.1.3.4, 168 U/mg), catalase (CAT from bovine liver, EC 1.11.1.6, 2,000-5,000 U/mg), peroxidase from horseradish (HRP, EC 1.11.1.7, 250-330 U/mg), flavin adenine dinucleotide (FAD disodium salt hydrate), $\beta$-Dglucose and DDAB were purchased from Sigma-Aldrich (Italy). GOD, HRP, CAT and FAD solutions $(100 \mu \mathrm{M})$ were prepared in $50 \mathrm{mM}$ phosphate buffer $\mathrm{pH} 7.4$ and stored at $4{ }^{\circ} \mathrm{C}$. $\beta$-Dglucose solution $(100 \mathrm{mM})$ was prepared in $50 \mathrm{mM}$ phosphate buffer $\mathrm{pH} 7.4$ and allowed to mutarotate at room temperature overnight before use. DDAB solution $(20 \mathrm{mM})$ was prepared in chloroform and stored at $4{ }^{\circ} \mathrm{C}$.

\subsection{Transmission electron microscopy (TEM)}

High-resolution images of GO in the presence of DDAB were collected with TEM (JEOL-3010 UHR, Jeol Ltd., Japan, operating at $300 \mathrm{keV}$ ) at room temperature. Specimens for TEM observation were prepared by casting one drop of $\mathrm{DDAB}$ chloroform solution and of GO water dispersion onto an amorphous carbon film supported on a copper mesh grid and drying in air. 


\subsection{Electrode preparation}

Before enzyme layer deposition, glassy carbon rotating disk electrodes (3 mm diameter, BASi, U.S.A) were polished with alumina and subsequently rinsed and sonicated in ultra-pure deionized water. Subsequently, glassy carbon electrodes were modified with $10 \mu \mathrm{L}$ of only $20 \mathrm{mM}$ DDAB chloroform solution or DDAB plus $5 \mu \mathrm{L}$ of GO water dispersion and then left at room temperature for 10 minutes to allow solvent evaporation. Five $\mu \mathrm{L}$ of GOD solution or free FAD solution were added and the modified glassy carbon electrodes were kept 2 hours at $4{ }^{\circ} \mathrm{C}$ before any further experimental procedure.

\subsection{Cyclic voltammetry and chronoamperometry}

All electrochemical experiments were carried out at room temperature $\left(25^{\circ} \mathrm{C}\right)$ and in $50 \mathrm{mM}$

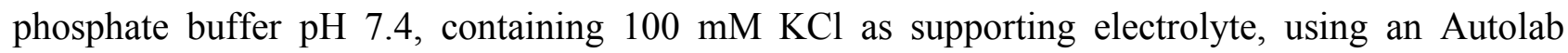
PGSTAT12 potentiostat (Ecochemie, The Netherlands) controlled by GPES3 software. A conventional three-electrode glass cell, equipped with a platinum wire counter electrode, an $\mathrm{Ag} / \mathrm{AgCl}(3 \mathrm{M} \mathrm{NaCl})$ reference electrode and $3 \mathrm{~mm}$ diameter glassy carbon working electrode (BASi, U.S.A), was also used.

Electrochemical investigation of GOD properties, both in the presence and in the absence of GO, was performed by cyclic voltammetry in a nitrogen atmosphere within a glovebox (Belle Technologies, U.K). Cyclic voltammograms were recorded between 0 and $-700 \mathrm{mV}$ (versus $\mathrm{Ag} / \mathrm{AgCl})$ at increasing scan rates.

Electrochemically driven $\beta$-D-glucose titration by GOD-DDAB-GO electrodes was performed using chronoamperometry with an applied potential bias of $600 \mathrm{mV}$.

In order to ensure the substrate permeation into the enzymatic layer and minimize mass transport influence at the transducer surface, GOD was immobilized through DDAB-GO on glassy carbon 
rotating disk electrodes. All chronoamperometric electrocatalysis experiments were performed with the rotational speed of $500 \mathrm{rpm}$ using a RDE-2 rotator system (BASi, U.S.A).

\section{Results and discussion}

\subsection{Transmission electron microscopy}

TEM images of $\mathrm{GO}$ in the presence of $\mathrm{DDAB}$ were collected in order to investigate the morphological features of GO layers distribution (Figure 1). As can be seen in the figure, GO is well distributed in a two-dimensional manner as a foil-like material, with exfoliated sheets characterized by wrinkling and waviness, in agreement with previously published experimental data $[17,22,26,27]$. Based on TEM results, it can be assumed that GO sheets are analogously distributed throughout the electrode surface and that DDAB-stabilized GOD molecules are directly interfacing the GO layer.

\subsection{Cyclic voltammetry}

Cyclic voltammetry of GOD was carried out using glassy carbon electrodes functionalized by either DDAB or DDAB-GO. Figure 2 shows cyclic voltammetry results obtained on GOD based DDAB electrodes both in the presence and in the absence of GO demonstrating the electrochemical reversibility of immobilized GOD, as revealed by a clearly defined reversible redox couple. Redox peak potential values of GOD-DDAB and GOD-DDAB-GO electrodes are summarized and compared to those of free FAD in Table 1. Interestingly as can be seen from the table, the presence of GO shifts the redox potential, calculated as midpoint potential $\left(\mathrm{E}_{1 / 2}\right)$, towards more positive values both for GOD bound and free FAD. The redox potential of the protein bound FAD also shifted slightly compared to free FAD, both in the presence and in the absence of GOD, however, these differences are not significant in electrochemical terms. In addition, no significant differences were found when comparing peak-to-peak separation values due to GO. Peak currents values were found to be linearly dependent on the scan rate, up to scan rate of $120 \mathrm{mVs}^{-1}$ (Figure 2, inset). As 
stated by Laviron's theory [28] this property is characteristic of thin film confined electroactive species that are not under diffusion control. A significant increase of the redox peak current values was also observed due to the presence of GO $(\mathrm{P}<0.001)$. Apparent surface coverage $(\Gamma)$ of the electroactive GOD bound and free FAD was calculated from the peak current plot versus scan rate by applying Faraday's law. As expected in the presence of GO the apparent surface coverage was higher both in the case of protein bound and free FAD. The statistical analysis performed by oneway ANOVA followed by Student-Newman-Keuls post hoc test also demonstrated that the coverage values calculated were significantly higher in the presence of GO (Table 1).

Investigation of the direct electrochemistry of GOD on DDAB-GO glassy carbon electrodes revealed an overall improvement of the enzyme's electrochemical features due to the presence of GO. In particular, the redox potential of both GOD bound and free FAD shifted to more positive values in the presence of GO therefore allowing the FAD to be reduced more readily by a less negative potential bias. GO also increased GOD peak currents, leading to an improvement in the signal-to-noise ratio. Moreover, an increase of the apparent surface coverage value was also observed proving that the number of electroactive enzyme molecules on the electrode surface are increased by the presence of GO.

\subsection{Electrochemical detection of $\beta$-D-glucose}

$\beta$-D-glucose was titrated on GOD based DDAB-GO electrodes used as first generation biosensor by which the amount of the enzymatically metabolized glucose is determined by the estimation of the hydrogen peroxide produced throughout reoxidation of fully reduced GOD (FAD hydroquinone) to oxidized GOD (FAD quinone) during the catalytic event [24]. Figure 3A shows the current responses upon $\beta$-D-glucose titration detected during chronoamperometric experiments, both in the presence and in the absence of GO. As can be seen, in the absence of GO there is a total suppression of the current response to $\beta$-D-glucose addition, proving that the capability to reoxidize hydrogen peroxide molecules (produced during the GOD turnover) is only possible in the presence of GO, as 
also reported in the literature [17]. Current signals detected upon $\beta$-D-glucose addition on DDABGO electrodes in the absence of GOD (negative control) were used to correct $\beta$-D-glucose titration experiments results on GOD-DDAB-GO electrodes. From the plot of the electrochemical responses to increasing amounts of $\beta$-D-glucose versus the $\beta$-D-glucose concentration (Figure $3 \mathrm{~B}$ ) the apparent kinetic parameters, $\mathrm{K}_{\mathrm{M}}{ }_{\mathrm{M}}$, indicator of enzyme-substrate reaction kinetics used to evaluate the biological activity of the immobilized enzyme, and $I_{\max }$, the maximum current detected under saturating substrate conditions, were estimated by fitting this plot with the Michaelis-Menten equation. The calculated values for $\mathrm{K}^{\prime}{ }_{\mathrm{M}}$ and $\mathrm{I}_{\max }$ were $1.04 \pm 0.12 \mathrm{mM}$ and $379.0 \pm 3.1 \mathrm{nA}$, respectively. The calculated apparent $\mathrm{K}_{M}$ value is in line with recently published results on GOD based $\beta$-D-glucose biosensors [29,30] and revealed a very high affinity of GOD-DDAB-GO for its substrate. In addition, the sensitivity of the system was estimated to be $220 \pm 7 \mu \mathrm{M}$ and calculated lower and upper limits of detection were $20 \mu \mathrm{M}$ and $600 \mu \mathrm{M}$ respectively, both parameters were estimated by the linear trace of the current plot versus $\beta$-D-glucose concentration.

In order to confirm that the signals measured upon $\beta$-D-glucose addition are due to hydrogen peroxide detection, a control experiment was performed by co-immobilizing GOD on DDAG-GO glassy carbon electrodes in the presence of horseradish peroxidase (HRP) or catalase (CAT). As expected, a significant impairment of current response to $\beta$-D-glucose addition was observed in the presence of HRP, whereas a total suppression of the current signals was observed when immobilizing GOD on DDAB-GO electrodes in the presence of CAT (Figure 4).

The inter-electrode reproducibility of the GOD-DDAB-GO system was also calculated by comparing the sensitivity of the response to $\beta$-D-glucose of several different electrodes. The electrode reproducibility, calculated as residual standard deviation, was found to be $3.2 \%$. 


\section{Conclusions}

Graphene oxide has many properties that can be useful for electrochemical biosensing. The use of $\mathrm{GO}$ in the presence of DDAB surfactant acting as a bio-membrane like microenvironment is here reported for the development of a GOD-based biosensor for the detection of $\beta$-D-glucose. The approach used for the construction of this GO based enzyme-electrode system is very rapid and reproducible. Direct electrochemistry of GOD was studied revealing an overall improved electrochemistry of this enzyme due to the presence of GO. Moreover, since glassy carbon electrode surfaces are not suitable for hydrogen peroxide measurements usually metallic electrode surfaces such as platinum or gold are used for the development of first generation biosensors that require long cleaning procedures. However, the addition of GO to glassy carbon electrodes was shown in this work to allow for $\beta$-D-glucose titrations on these surfaces, achieved through the detection of hydrogen peroxide (which is formed during the catalysis of GOD).

This fabricated enzyme-electrode system showed excellent electrochemical behavior where the direct electron transfer between the GOD and the modified electrode was easily achieved and, most importantly, the detection of $\beta$-D-glucose was successfully carried out showing a good sensitivity and a very high affinity of the substrate with the GO based enzyme electrode. Taken together, the simplicity and ease of the electrode preparation procedure of the GO/DDAB system make it a good candidate for immobilization of other biomolecules for amperometric devices such as biosensors.

\section{ACKNOWLEDGMENT}

The authors wish to acknowledge financial support from the Progetto Ateneo-San Paolo 2012.

\section{Notes}

The authors declare no competing financial interest. 


\section{References}

[1] He, H.Y., Riedl, T., Lerf, A., Klinowski, J. (1996) J. Phys. Chem. 100, 19954-19958.

[2] Lerf, A., He, H., Forster, M., Klinowski, J. (1998) J. Phys. Chem. B 102, 4477-4482.

[3] He, H.Y., Klinowski, J., Forster, M., Lerf, A. (1998) Chem. Phys. Lett. 287, 53-56.

[4] Compton, O.C., Nguyen, S.T. (2010) Small 6, 711-723.

[5] McCreery, R.L. (2008) Chem. Rev. 108, 2646-2687.

[6] Shan, C., Yang, H., Song, J., Han, D., Ivaska, A., Niu, L. (2009) Anal. Chem. 81, 2378-2382.

[7] Banks, C.E., Davies, T.J., Wildgoose, G.G., Compton, R.G. (2005) Chem. Commun. (Camb). 7, 829-841.

[8] Galande, C., Gao, W., Mathkar, A., Dattelbaum, A.M., Narayanan, T.N., Mohite, A.D., Ajayan, P.M. (2014) Part. Part. Syst. Charact. 31, 619-638.

[9] Dreyer, D.R., Todd, A.D., Bielawski, C.W. (2014) Chem. Soc. Rev. 43, 5288-5301.

[10] Pumera, M. (2011) Mater. Today 14, 308-315.

[11] Artiles, M.S., Rout, C.S., Fisher, T.S. (2011) Adv. Drug. Deliv. Rev. 63, 1352-1360.

[12] Kuila, T., Bose, S., Khanra, P., Mishra, A.K., Kim, N.H., Lee, J.H. (2011) Biosens. Bioelectron. 26, 4637-4648.

[13] Liu, Y., Yu, D., Zeng, C., Miao, Z., Dai, L. (2010) Langmuir 26, 6158-6160.

[14] Hong, B.J., An, Z., Compton, O.C., Nguyen, S.T. (2012) Small 8, 2469-2476.

[15] Morales-Narváez, E., Merkoçi, A. (2012) Adv. Mater. 24, 3298-3308.

[16] Zhu, Y., Murali, S., Cai, W., Li, X., Suk, J.W., Potts, J.R., Ruoff, R.S. (2010) Adv. Mater. 22, 3906-3924.

[17] Zhou, M., Zhai, Y., Dong, S. (2009) Anal. Chem. 81, 5603-5613.

[18] Shao, Y., Wang, J., Engelhard, M., Wang, C., Lin, Y. (2010) J. Mat. Chem. 20, 743-748.

[19] Rusling, J.F. (1998) Acc. Chem. Res. 31, 363-369. 
[20] Sadeghi, S.J., Meirinhos, R., Catucci, G., Dodhia, V.R., Di Nardo, G., Gilardi, G. (2010) J. Am. Chem. Soc. 132,458-459.

[21] Castrignanò, S., Sadeghi, S.J., Gilardi, G. (2012) Biochim. Biophys. Acta 1820, 2072-2078.

[22] Castrignanò, S., Gilardi, G., Sadeghi, S.J. (2015) Anal. Chem. DOI: 10.1021/ac504535y.

[23] Cui, D., Mi, L., Xu, X., Lu, J., Qian, J., Liu, S. (2014) Langmuir 30, 11833-11840.

[24] Zhu, Z., Garcia-Gancedo, L., Flewitt, A.J., Xie, H., Moussy, F., Milne, W.I. (2012) Sensors (Basel). 12, 5996-6022.

[25] Song, Y., Qu, K., Zhao, C., Ren, J., Qu, X. (2010) Adv. Mater. 22, 2206-2210.

[26] Dikin, D.A., Stankovich, S., Zimney, E.J., Piner, R.D., Dommett, G.H., Evmenenko, G., Nguyen, S.T., Ruoff, R.S. (2007) Nature 448, 457-460.

[27] Wang, K.; Ruan, J.; Song, H.; Zhang, J.; Wo, Y.; Guo, S.; Cui, D. (2011) Nanoscale Res. Lett. 6, 8-15.

[28] Laviron, E.J. (1979) Electroanal. Chem. 101, 19-28.

[29] Wang, K., Liu, Q., Guan, Q.M., Wu, J., Li, H.N., Yan, J.J. (2011) Biosens. Bioelectron. 26, $2252-2257$.

[30] Zhou, K.F., Zhu, Y.H., Yang, X.L., Li, C.Z. (2010) Electroanal. 22, 259-264. 
Table 1. Redox properties of GOD in the presence or absence of GO compared to those of free FAD.

\begin{tabular}{llrl}
\hline & \multicolumn{1}{c}{$\mathbf{E}_{\mathbf{1} / \mathbf{2}} / \mathbf{m V}$} & $\Delta \mathbf{E} / \mathbf{~ m V}$ & $\Gamma / \mathbf{p m o l ~ c m}^{-\mathbf{2}}$ \\
\hline GC/GOD-DDAB & $-367.4 \pm 0.2$ & $53.0 \pm 2.6$ & $21.2 \pm 2.4$ \\
\hline GC/GOD-DDAB-GO & $-337.1 \pm 2.1^{(* *)}$ & $53.4 \pm 2.9$ & $43.7 \pm 1.8^{(* *)}$ \\
\hline GC/FAD-DDAB & $-353.7 \pm 3.5^{(\#)}$ & $50.7 \pm 9.6$ & $27.6 \pm 0.2$ \\
\hline GC/FAD-DDAB-GO & $-344.3 \pm 4.0^{(*, \#)}$ & $49.3 \pm 1.5$ & $64.9 \pm 6.9^{(* *)}$
\end{tabular}

(*) $\mathrm{P}<0.05,(* *) \mathrm{P}<0.001$ when comparing data obtained in the absence of GO. $\left(^{\#}\right) \mathrm{P}<0.05$ when comparing data obtained with GOD protein. 


\section{Figure captions}

Figure 1: TEM image of GO in the presence of DDAB.

Figure 2: Anaerobic cyclic voltammograms of GOD on DDAB glassy carbon electrodes in the absence (thin line) and in the presence (bold line) of GO. Scan rate: $120 \mathrm{mV} \mathrm{s}^{-1}$. Inset: plot of cathodic (filled symbols) and anodic (empty symbols) peak currents versus scan rate for GOD on DDAB glassy carbon electrodes in the absence (triangles) and in the presence (circles) of GO. $\mathrm{R}^{2}>$ 0.99 .

Figure 3: A) Amperometric response upon $\beta$-D-glucose addition on GOD-DDAB glassy carbon electrodes in the presence (bold line) and in the absence (thin line) of GO. Arrows indicate addition of $0.2 \mathrm{mM} \beta$-D-glucose to the electrolysis cell. B) Corrected current signals measured upon $\beta$-Dglucose titration on GOD-DDAB-GO electrodes plotted against $\beta$-D-glucose concentration fitted to a Michaelis-Menten equation. Data are mean \pm standard deviation of five different biological replicates. $\mathrm{R}^{2}>0.99$.

Figure 4: Amperometric response upon $\beta$-D-glucose addition on GOD-DDAB-GO (A), GODHRP-DDAB-GO (B) and GOD-CAT-DDAB-GO (C) glassy carbon electrodes. Arrows indicate addition of $0.2 \mathrm{mM} \beta$-D-glucose to the electrolysis cell. 